\title{
On the Hilbert-Samuel Function
}

\author{
Kazuji KUBOTA \\ The National Defense Academy \\ (Communicated by Y. Kawada)
}

\section{Introduction}

Let $A$ be a Cohen-Macaulay semi-local ring of dimension $d$. The length of an $A$-module $E$ will be denoted by $\ell(E)$. Let $I$ be an open ideal of $A$ which contains some power of the Jacobson radical of $A$, then the Hilbert-Samuel function $\ell\left(A / I^{n+1}\right)$ of $I$ equals

$$
e_{0}\left(\begin{array}{c}
n+d \\
d
\end{array}\right)-e_{1}\left(\begin{array}{c}
n+d-1 \\
d-1
\end{array}\right)+\cdots+(-1)^{d-1} e_{d-1}\left(\begin{array}{c}
n+1 \\
1
\end{array}\right)+(-1)^{d} e_{d}
$$

for large $n$. The coefficients $e_{k}(0 \leqq k \leqq d)$ are called the normalised HilbertSamuel coefficients of $I . \quad e_{k}$ will be denoted by $e_{k}(I)$, if it is necessary to avoid confusion.

Assume that $A$ is a Cohen-Macaulay local ring of dimension $d>0, M$ the maximal ideal of $A$, and the residue field $A / M$ infinite. Abhyankar [1] proved inequality

$$
\iota\left(M / M^{2}\right) \leqq e+d-1
$$

where $e=e_{0}(M)$. Sally [11] proved that equality holds if and only if there exists an ideal $X$ generated by a system of parameters of $A$ such that $M^{2}=X M$, and that if equality holds then

$$
\measuredangle\left(A / M^{n+1}\right)=e\left(\begin{array}{c}
n+d-1 \\
d
\end{array}\right)+\left(\begin{array}{c}
n+d-1 \\
d-1
\end{array}\right)
$$

for all $n \geqq 0$ ([12] Theorem 1 ). We know also

$$
\ell\left(M / M^{2}\right)=e+d-1-\ell\left(M^{2} / X M\right)
$$

for any ideal $X$ generated by a system of parameters of $A$, such that $M^{n+1}=X M^{n}$ for large $n$ ([13] Lemma 2.1). Furthermore, for a primary 
ideal $I$ which belongs to the maximal ideal $M$, Valla [15] obtained equality

$$
\iota\left(I / I^{2}\right)=e+(d-1) \zeta(A / I)-\ell\left(I^{2} / X I\right)
$$

where $e=e_{0}(I)$ and $X$ is an ideal generated by a system of parameters of $I$ which is contained in $I$ and satisfies $e=\ell(A / X)$.

Let $A$ be again, a Cohen-Macaulay semi-local ring, and $I$ an open ideal of $A$. We denote a ring of fractions $S^{-1} A$ by $A_{I}$, where $S$ is the set of all elements of $A$ which are contained in no prime divisors of $I$. If a system of $d$ elements of $I$ generates an ideal which contains some power of $I$, we call it a system of parameters of $I$. In this paper, we shall put the above results of precursors together to the following synthetical theorem.

Theorem. Let $A$ be an equi-dimensional Cohen-Macaulay semi-local ring of dimension $d>0$, and $I$ an open ideal of $A$. Then the following conditions are equivalent.

(1) $\angle\left(A / I^{n+1}\right)=e_{0}\left(\begin{array}{c}n+d \\ d\end{array}\right)-e_{1}\left(\begin{array}{c}n+d-1 \\ d-1\end{array}\right)$ for all $n \geqq 0$.

(2) $\angle(A / I)=e_{0}-e_{1}$ and $\angle\left(A / I^{2}\right)=e_{0}(d+1)-e_{1} d$.

(3) $\ell\left(A / I^{2}\right)=e_{0}+d \ell(A / I)$.

If the residue fields $A / M$ are infinite for all maximal ideals $M$ of $A$, then the above conditions are equivalent to the next one.

(4) There exists an ideal $X$ in $A_{I}$, generated by a system of parameters of $I A_{I}$ such that $I^{2} A_{I}=X I A_{I}$.

As a corollary to the theorem, we have a new proof of a result on $e_{2}$ obtained by Narita [7].

Sally [14] proved that a Cohen-Macaulay local ring with its maximal ideal $M$ and multiplicity $e$, has the maximal embedding dimension $e+d-1$, if and only if its Hilbert-Samuel polynomial is

$$
e\left(\begin{array}{c}
n+d-1 \\
d
\end{array}\right)+\left(\begin{array}{c}
n+d-1 \\
d-1
\end{array}\right)
$$

We shall show in a forthcoming paper [3], an extension of this fact to any open ideal of an equi-dimensional Cohen-Macaulay semi-local ring.

In our terminology, a ring $A$ is said equi-dimensional, if $\operatorname{dim}\left(A_{\mu}\right)=$ $\operatorname{dim}(A)$ for all maximal ideals $M$ of $A$. Thus, an equi-dimensional CohenMacaulay ring is a Macaulay ring of Nagata [6]. Throughout the paper, we assume that $A$ is an equi-dimensional Cohen-Macaulay semi-local ring of dimension $d>0$. An element of $A$ which is not a zero-divisor will be called a regular element. 
I should like to express my thanks to Prof. Y. Kawada for his encouragement during the preparation of this paper, and I am indebted to T. Watanabe for his inspiring suggestions.

\section{§1. One-dimensional case.}

In this section, we assume that the dimension $d$ of $A$ is equal to 1 , and we recall that an open ideal of $A$ which satisfies the equivalent conditions in the theorem, is nothing but a stable ideal studied by Lipman [4].

Let $\bar{A}$ be the integral closure of $A$ in its total ring of fractions, and put $I^{n}: I^{n}=\left\{x \in \bar{A} \mid x I^{n} \subset I^{n}\right\}$ for an open ideal $I$ of $A$. $A$ ring $A^{I}=$ $\cup_{n>0} I^{n}: I^{n}$ is called the blowing-up of $A$ with center $I$. We know that $A^{I}=I^{n}: I^{n}$ for large $n$ and $A^{I}$ is a finite $A$-algebra. Furthermore $I A^{I}$ is a principal ideal of $A^{I}$ generated by a regular element. An open ideal $I$ is called a stable ideal, if $I A^{I}=I$ or $A^{I}=I: I$. For large $n, I^{n}$ is stable, since the blowing-up of $A$ with center $I^{n}(n \geqq 1)$ coincides with $A^{I}$. If $I^{r}$ $(r \geqq 1)$ is stable, then $I^{n}$ are stable for all $n \geqq r$. An element $x$ of $I$ is called a transversal element of $I$, if $I^{n+1}=x I^{n}$ for large $n$. If the residue fields $A / M$ are infinite for all maximal ideals $M$ of $A$, then there exists a transversal element of $I$. Let $x$ be a transversal element of $I$, then $I A^{I}=x A^{I}$ and $I^{n}$ is stable if and only if $I^{n+1}=x I^{n}$. Now put $e=\ell\left(A^{I} / I A^{I}\right)$ and $f=\ell\left(A^{I} / A\right)$. Since, if $x$ is a regular element in $A, \ell(E / x E)=\ell(A / x A)$ for any finite $A$-module $E$ in $\bar{A}$, we have $e=\ell(A / x A)$ if $x$ is transversal element of $I$.

Proposition 1. For an open ideal I of $A$, we have the following inequalities.

(1) $\measuredangle\left(A / I^{n}\right) \geqq e n-f$ for all $n \geqq 0$, and for $n \geqq 1$ equality holds if and only if $I^{n}$ is stable. In particular, $e=e_{0}(I)$ and $f=e_{1}(I)$ ([4] Theorem 1.5 or [5] Propositon 9).

(2) $\ell\left(I^{n} / I^{n+1}\right) \leqq e$ for all $n \geqq 0$, and for $n \geqq 1$ equality holds if and only if $I^{n}$ is stable ([4] Theorem 1.9 or [5] Proposition 8).

Proof. Since $I A^{I}$ is a principal ideal of $A^{I}$ generated by a regular element, $\iota\left(I^{k} A^{I} / I^{k+1} A^{I}\right)=\ell\left(A^{I} / I A^{I}\right)=e$ for all $k \geqq 0$, and

$$
\iota\left(A^{I} / I^{n} A^{I}\right)=\iota\left(A^{I} / I A^{I}\right)+\cdots+\iota\left(I^{n-1} A^{I} / I^{n} A^{I}\right)=e n
$$

for $n \geqq 0$. Therefore,

$$
\iota\left(A / I^{n}\right)=\iota\left(A^{I} / I^{n}\right)-\ell\left(A^{I} / A\right)
$$




$$
\begin{aligned}
& =\ell\left(A^{I} / I^{n} A^{I}\right)+\ell\left(I^{n} A^{I} / I^{n}\right)-\ell\left(A^{I} / A\right) \\
& =e n-f+\ell\left(I^{n} A^{I} / I^{n}\right) .
\end{aligned}
$$

This proves (1). To prove (2), consider $A[t]_{I_{L}[t]}$ where $t$ is an indeterminate, if necessary, and we may assume that $A / M$ are infinite fields for all maximal ideals $M$ of $A$ (cf. [4] p. 656, [5] p. 277, or [6] (21.4)). Then there exists a transversal element $x$ of $I$, and $\ell\left(I^{n} / x I^{n}\right)=\ell(A / x A)=e$ for all $n \geqq 0$. Hence,

$$
\ell\left(I^{n} / I^{n+1}\right)=\ell\left(I^{n} / x I^{n}\right)-\ell\left(I^{n+1} / x I^{n}\right)=e-\ell\left(I^{n+1} / x I^{n}\right),
$$

and we obtain (2).

COROLlary 2. The following conditions for an open ideal I of A, are equivalent.

(1) I is stable.

(2) $\angle(A / I)=e-f$.

(3) $\angle\left(A / I^{n}\right)=e n-f$ for all $n \geqq 1$.

(4) $\ell\left(I / I^{2}\right)=e$.

(5) $\angle\left(I^{n} / I^{n+1}\right)=e$ for all $n \geqq 1$.

(6) There exists an element $x$ of $I$ such that $I^{2}=x I$.

Proof. There exists an element $x$ of $A^{I}$ such that $I A^{I}=x A^{I}$. Assume (1), then $I=I A^{I}=x A^{I}$ and $x$ is an element of $I$. Moreover, $I^{2}=I^{2} A^{I}=$ $x I A^{I}=x I$, and we have (6). The rest of the proof is obvious.

\section{§2. Systems of superficial parameters.}

Hereafter, we assume that the dimension of $A d>0$, and for any subsets $J$ and $K$ of $A$, we put $J: K=\{x \in A \mid x K \subset J\}$. An element $x$ of an open ideal $I$ is called a superficial element of $I$, if there exists an integer $c$ such that $\left(I^{n+1}: x\right) \cap I^{\circ}=I^{n}$ for all $n \geqq c$. We call an open ideal contained in the Jacobson radical of $A$, an ideal of definition of $A$. If $I$ is an open ideal of $A$, then $I A_{I}$ is an ideal of definition of $A_{I} . A$ system of parameters of an ideal of definition, is a regular sequence in $A([6] \S 25)$.

LEMMA 3. An element $x$ of an ideal of definition $I$, is a superficial element of $I$ if and only if $I^{n+1}: x=I^{n}+(0: x)$ and $I^{n} \cap(0: x)=0$ for all large $n$.

Proof. Assume that the conditions in the lemma are satisfied by $n \geqq c$. Then $\left(I^{n+1}: x\right) \cap I^{o}=I^{n}+(0: x) \cap I^{c}=I^{n}$ for $n \geqq c$. Therefore $x$ is a superficial element of $I$. 
Conversely assume that $x$ is a superficial element of $I$, and $\left(I^{n+1}: x\right) \cap$ $I^{\circ}=I^{n}$ for all $n \geqq c$. By the Artin-Rees lemma, there exists an integer $k$ such that $I^{n} \cap x A=I^{n-k}\left(I^{k} \cap x A\right)$ for all $n \geqq k$. Hence for $n \geqq c+k-1, I^{n+1} \cap$ $x A$ is contained in $x I^{c}$, and we have

$$
I^{n+1}: x=\left(I^{n+1}: x\right) \cap I^{\circ}+(0: x)=I^{n}+(0: x) .
$$

On the other hand if $n \geqq c$ and $m \geqq c$, then we have

$$
I^{n} \cap(0: x) \subset I^{c} \cap(0: x) \subset I^{c} \cap\left(I^{m+1}: x\right)=I^{m} .
$$

Hence by Krull's Intersection Theorem, $I^{n} \cap(0: x)=0$ for $n \geqq c$.

LEMMA 4. Let $I$ be an ideal of definition of $A$.

(1) An element $x$ of $I$ is a superficial element of $I$, if and only if $I^{n+1}: x=I^{n}$ for all large $n$. In particular, any superficial element of $I$ is a regular element.

(2) Let $x$ be a superficial element of $I$, and $n(\geqq 0)$ an integer. Then we have equality

$$
\measuredangle\left(A /\left(I^{n+1}+x A\right)\right)=\iota\left(A / I^{n+1}\right)-\ell\left(A / I^{n}\right),
$$

if and only if $I^{n+1}: x=I^{n}$.

(3) If $x$ is a superficial element of I, then we have

$$
e_{k}(I)=e_{k}(I / x A)
$$

for all $k(0 \leqq k \leqq d-1)$.

Proof. Let $x$ be an element of $I$, then

$$
\begin{aligned}
\iota\left(A /\left(I^{n+1}+x A\right)\right) & =\ell\left(A / I^{n+1}\right)-\ell\left(\left(I^{n+1}+x A\right) / I^{n+1}\right) \\
& =\measuredangle\left(A / I^{n+1}\right)-\ell\left(x A /\left(I^{n+1} \cap x A\right)\right) \\
& =\measuredangle\left(A / I^{n+1}\right)-\ell\left(A /\left(I^{n+1}: x\right)\right) \\
& =\measuredangle\left(A / I^{n+1}\right)-\ell\left(A / I^{n}\right)+\ell\left(\left(I^{n+1}: x\right) / I^{n}\right) .
\end{aligned}
$$

If $x$ is a superficial element of $I$, then the above Lemma 3 implies that the inclusion $0: x \subset I^{n+1}: x$ induces an isomorphism $0: x \cong\left(I^{n+1}: x\right) / I^{n}$ for large $n$. Therefore

$$
\zeta\left(A /\left(I^{n+1}+x A\right)\right)=\iota\left(A / I^{n+1}\right)-\iota\left(A / I^{n}\right)+\zeta(0: x)
$$

for all large $n$. Since there exists a superficial element of $I$ which is regular, the above equality implies that the degree of the Hilbert-Samuel function $\angle\left(A / I^{n+1}\right)$ is equal to the dimension $d$ of $A$. For any superficial 
element $x$ of $I$, then the degree of the Hilbert-Samuel function $\zeta\left(A /\left(I^{n+1}+x A\right)\right)$ of $I / x A$ is $d-1$ and we know that $\operatorname{dim}(A / x A)=d-1$. Since $A$ is an equi-dimensional Cohen-Macaulay semi-local ring, and $I$ is an ideal of definition of $A$, this means that $x$ is a regular element. Hence we have (1). (2) and (3) are immediate consequences of (1).

LEMMA 5. Let $x_{1}, x_{2}, \cdots, x_{t}, x$ be a regular sequence in the Jacobson radical of $A$, and $X=\left(x_{1}, x_{2}, \cdots, x_{t}\right)$. Then $X^{n}: x=X^{n}$ for all $n \geqq 0$.

Proof. We prove the assertion by induction on $n$. The assertion for $n=0$ is trivial, and we assume that $n \geqq 1$. By hypothesis of induction, $X^{n-1}=X^{n-1}: x \supset X^{n}: x$. We prove

$$
\left(x_{1}, \cdots, x_{s}\right)^{n-1} \cap\left(X^{n}: x\right) \subset X^{n}
$$

for all $s(0 \leqq s \leqq t)$, again by induction on $s$. For $s=0$, we take $\left(x_{1}, \cdots, x_{s}\right)=0$ and the assertion is obvious. Now let $s \geqq 1$, and $w$ an element of $\left(x_{1}, \cdots, x_{s}\right)^{n-1} \cap\left(X^{n}: x\right)$. We can put $w=u+x_{s} v$, where $u$ and $v$ are elements of $\left(x_{1}, \cdots, x_{s-1}\right)^{n-1}$ and $\left(x_{1}, \cdots, x_{s}\right)^{n-2}$ respectively. Let $Y=\left(x_{1}, \cdots, x_{s-1}, x_{s+1}, \cdots, x_{t}\right)$, then $x w=x u+x x_{s} v$ is contained in $X^{n}=Y^{n}+$ $x_{8} X^{n-1}$. And we can find an element $z$ of $X^{n-1}$ such that $x w-x_{8} z=x u+$ $x_{s}(x v-z)$ is an element of $Y^{n}$. Since $u \in Y^{n-1}$, we get $x_{s}(x v-z) \in Y^{n-1}$. Therefore by hypothesis of induction on $n$, we have $x v-z \in Y^{n-1}$ and $x v \in X^{n-1}$. Again by hypothesis of induction on $n$, we know that $v \in X^{n-1}$. Hence $x u=x w-x x_{s} v \in X^{n}$, and $u$ is contained in $\left(x_{1}, \cdots, x_{s-1}\right)^{n-1} \cap\left(X^{n}: x\right)$. By hypothesis of induction on $s, u \in X^{n}$, and we know that $w=u+x_{s} v$ is contained in $X^{n}$.

We call a sequence of $d$ elements of $I, x_{1}, x_{2}, \cdots, x_{d}$, a system of superficial parameters of $I$, if $x_{k} \bmod \left(x_{1}, \cdots, x_{k-1}\right)$ is a superficial element of $I /\left(x_{1}, \cdots, x_{k-1}\right)$ for any $k(1 \leqq k \leqq d)$. A system of superficial parameters of an ideal of definition $I$, is a system of parameters of $I$.

LEMma 6. Let $X$ be an ideal generated by a system of parameters of an ideal of definition $I, x_{1}, x_{2}, \cdots, x_{d}$. Put $I_{k}=I /\left(x_{1}, \cdots, x_{k-1}\right)$ for each $k(1 \leqq k \leqq d)$. Then the following conditions are equivalent.

(1) $I^{2}=X I$.

(2) $I^{2} \subset X$, and $\left(I_{k}\right)^{n+1}: x_{k}=\left(I_{k}\right)^{n}$ for all $k(1 \leqq k \leqq d)$ and for all $n \geqq 0$. Where we confound $x_{k}$ with its image in $A /\left(x_{1}, \cdots, x_{k-1}\right)$.

Proof. Assume (1) and put $Y=\left(x_{2}, \cdots, x_{d}\right)$. Then $I^{n+1}=x_{1} I^{n}+Y^{n} I$. Let $w \in I^{n+1}: x_{1}$ and $x_{1} w=x_{1} u+v$ where $u \in I^{n}$ and $v \in Y^{n} I$, then $x_{1}(w-u) \in Y^{n}$. Since $x_{2}, \cdots, x_{d}, x_{1}$ is a regular sequence in $A$, we have $w-u \in Y^{n}=Y^{n}: x_{1}$ by the preceding Lemma 5 . Therefore $w$ is contained in $I^{n}$, and $I^{n+1}: x_{1}=I^{n}$. 
Since $\left(I_{k}\right)^{2}=\left(x_{k}, \cdots, x_{d}\right) I_{k}$, we have $\left(I_{k}\right)^{n+1}: x_{k}=\left(I_{k}\right)^{n}$ for any $k(1 \leqq k \leqq d)$. This proves (2). On the other hand, (2) obviously implies (1). In general, if for an integer $n \geqq 0, I^{n+1}$ is contained in $X=\left(x_{1}, x_{2}, \cdots, x_{d}\right)$ where $x_{k}$ $(1 \leqq k \leqq d)$ are elements of $I$, and if $\left(I_{k}\right)^{n+1}: x_{k}=\left(I_{k}\right)^{n}$ for all $k(1 \leqq k \leqq d)$, then we have $I^{n+1}=X I^{n}$.

\section{§3. Proof of Theorem.}

Let $I$ be an open ideal of an equi-dimensional Cohen-Macaulay semilocal ring $A$ of dimension $d>0$. Since $A / I$ is canonically isomorphic to $A_{I} / I A_{I}$, the length of an $A$-module $I^{n} / I^{n+1}$ equals to the length of an $A_{I^{-}}$ module $\left(I A_{I}\right)^{n} /\left(I A_{I}\right)^{n+1}$. Therefore, the Hilbert-Samuel function of $I$ and that of $I A_{I}$ take the same value for any $n \geqq 0$, and $e_{k}(I)=e_{k}\left(I A_{I}\right)$ for all $k(0 \leqq k \leqq d)$.

Proposition 7 (cf. [9] Theorem 1). For an open ideal $I$ of $A$, we have inequality

$$
e_{1} \geqq e_{0}-\ell(A / I) \geqq 0 \text {. }
$$

Proof. First, consider $A_{I}$ if necessary, and we may assume that $I$ is an ideal of definition. Next, consider $A[t]_{I A[t]}$ with an indeterminate $t$ if necessary, we may assume that $A / M$ are infinite fields for all maximal ideals $M$ of $A$. Then there exists a system of superficial parameters of $I, x_{1}, x_{2}, \cdots, x_{d}$, and we have $e_{k}(I)=e_{k}\left(I /\left(x_{1}, \cdots, x_{d-1}\right)\right)$ for $k=0$ and $k=1$ by (3) of Lemma 4. Hence the assertion is obtained by Proposition 1.

Proposition 8 (cf. [15] Lemma 1). For an open ideal $I$ of $A$, we have inequality

$$
\iota\left(I / I^{2}\right) \leqq e_{0}+(d-1) \zeta(A / I) .
$$

If $X$ is an ideal of $A_{I}$ generated by a system of parameters of $I A_{I}$ such that $e_{0}=\iota_{A_{T}}\left(A_{I} / X\right)$, then we have equality

$$
\iota\left(I / I^{2}\right)=e_{0}+(d-1) \zeta(A / I)-\iota_{A_{I}}\left(I^{2} A_{I} / X I A_{I}\right) .
$$

Proof. Again, we may assume that $I$ is an ideal of definition and that $A / M$ are infinite fields for all maximal ideals $M$ of $A$. Let $x_{1}, x_{2}, \cdots, x_{d}$ be a system of superficial parameters of $I$, then $e_{0}(I)=$ $\measuredangle\left(A /\left(x_{1}, x_{2}, \cdots, x_{d}\right)\right)$ by (3) of Lemma 4 and by the fact that $x_{d}$ mod. $\left(x_{1}, \cdots, x_{d-1}\right)$ is a transversal element of $I /\left(x_{1}, \cdots, x_{d-1}\right)$. In general, if $X$ is an ideal generated by a system of parameters of $I, x_{1}, x_{2}, \cdots, x_{d}$, then $x_{1}, x_{2}, \cdots, x_{d}$ is a regular sequence in $A$. Therefore, a homomorphism $\phi$ from a direct product of $d$ copies of $A$ to $X / \dot{X} I$ defined by 


$$
\phi\left(a_{1}, a_{2}, \cdots, a_{d}\right)=a_{1} x_{1}+a_{2} x_{2}+\cdots+a_{d} x_{d} \bmod . X I,
$$

induces an isomorphism from a direct product of $d$ copies of $A / I$ to $X / X I$. Hence we have $\iota(X / X I)=d \iota(A / I)$, and

$$
\begin{aligned}
\ell\left(I / I^{2}\right) & =\zeta\left(A / I^{2}\right)-\ell(A / I) \\
& =\zeta(A / X)+\zeta(X / X I)-\iota\left(I^{2} / X I\right)-\ell(A / I) \\
& =\zeta(A / X)+(d-1) \zeta(A / I)-\ell\left(I^{2} / X I\right) .
\end{aligned}
$$

This proves the proposition.

Now, we come to prove our Theorem.

Proof of Theorem. Obviously (1) implies (2), and (2) does (3). To prove that (3) implies (1), we may assume as usual, that $I$ is an ideal of definition and that the residue fields $A / M$ are infinite for all maximal ideals $M$ of $A$. Under this condition, there exists an ideal $X$ generated by a system of superficial parameters of $I$. Since $e_{0}(I)=\bullet(A / X)$, (3) implies (4) by Proposition 8. Now assume (4), and let $X$ be an ideal generated by a system of parameters of $I, x_{1}, x_{2}, \cdots, x_{d}$ such that $I^{2}=X I$. For $k(1 \leqq k \leqq d)$ put $X_{k}=\left(x_{1}, \cdots, x_{k-1}\right), A_{k}=A / X_{k}$, and $I_{k}=I / X_{k}$. Then by Lemma 6, $\left(I_{k}\right)^{n+1}: x_{k}=\left(I_{k}\right)^{n}$ for all $n \geqq 0$, where we again confound $x_{k}$ with its image in $A_{k}$. Therefore by (2) of Lemma 4, we have

$$
\zeta\left(A_{k} /\left(I_{k}\right)^{n+1}\right)-\ell\left(A_{k} /\left(I_{k}\right)^{n}\right)=\ell\left(A_{k+1} /\left(I_{k+1}\right)^{n+1}\right)
$$

for all $k(1 \leqq k \leqq d-1)$ and for all $n \geqq 0$. Since $\left(I_{d}\right)^{2}=x_{d} I_{d}, I_{d}$ is a stable ideal of a one-dimensional ring $A_{d}$. Therefore by Corollary 2, we have

$$
\iota\left(A_{d} /\left(I_{d}\right)^{n+1}\right)=e_{0}\left(I_{d}\right)(n+1)-e_{1}\left(I_{d}\right)
$$

for all $n \geqq 0$. Since $e_{k}\left(I_{d}\right)=e_{k}(I)=e_{k}$ for $k=0$ and $k=1$, we have

$$
C\left(A / I^{n+1}\right)=e_{0}\left(\begin{array}{c}
n+d \\
d
\end{array}\right)-e_{1}\left(\begin{array}{c}
n+d-1 \\
d-1
\end{array}\right)
$$

for all $n \geqq 0$. This completes the proof of the Theorem.

Corollary 9 (cf. [7] Theorem 1). Assume that the dimension $d \geqq 2$, then for an open ideal $I$, we have $e_{2} \geqq 0$. In the case that $d=2$ and that $A / M$ are infinite fields for all maximal ideals $M$ of $A, e_{2}=0$ if and only if there exists an integer $r(\geqq 1)$ and an ideal $X$ in $A_{I}$ generated by a system of parameters of $I^{r} A_{I}$ such that $I^{2 r} A_{I}=X I^{r} A_{I}$.

Proof. For an integer $r(\geqq 1)$; let 


$$
P_{r}(n)=e_{0}\left(I^{r}\right)\left(\begin{array}{c}
n+d \\
d
\end{array}\right)-e_{1}\left(I^{r}\right)\left(\begin{array}{c}
n+d-1 \\
d-1
\end{array}\right)+\cdots+(-1)^{d} e_{d}\left(I^{r}\right)
$$

be the Hilbert-Samuel polynomial of $I^{r}$. We can write

$$
P_{r}(n)=(n+1) Q_{r}(n)+(-1)^{d} e_{d}\left(I^{r}\right),
$$

where $Q_{r}(n)$ is a polynomial in $n$. For large $n$, the Hilbert-Samuel function of $I^{r}, \ell\left(A / I^{r(n+1)}\right)$ equals to both $P_{r}(n)$ and $P_{1}(r(n+1)-1)$, and we have equality of polynomials

$$
P_{r}(n)=r(n+1) Q_{1}(r(n+1)-1)+(-1)^{d} e_{n}(I) .
$$

Putting $n=-1$, we get $e_{d}\left(I^{r}\right)=e_{d}(I)$ for any integer $r(\geqq 1)$ ([7] Proposition 2).

To prove $e_{2}=e_{2}(I) \geqq 0$, we may assume again, that $I$ is an ideal of definition and that $A / M$ are infinite fields for all maximal ideals $M$ of $A$. Further we may assume that $d=2$, by (3) of Lemma 4 . Let $r(\geqq 1)$ be an integer such that

$$
\iota\left(A / I^{n+1}\right)=e_{0}\left(\begin{array}{c}
n+2 \\
2
\end{array}\right)-e_{1}\left(\begin{array}{c}
n+1 \\
1
\end{array}\right)+e_{2}
$$

for all $n \geqq r-1$. Put $J=I^{r}$, then

$$
\iota\left(A / J^{n+1}\right)=e_{0}(J)\left(\begin{array}{c}
n+2 \\
2
\end{array}\right)-e_{1}(J)\left(\begin{array}{c}
n+1 \\
1
\end{array}\right)+e_{2}(J)
$$

for all $n \geqq 0$. In particular putting $n=0$, we get

$$
e_{2}=e_{2}(I)=e_{2}(J)=e_{1}(J)-e_{0}(J)+\ell(A / J) \geqq 0,
$$

by Proposition 7. This proves the first assertion of the corollary. If $d=2$ and $e_{2}=0$, then the above $J=I^{r}$ satisfies the condition (1) of Theorem. Therefore, equivalence of (1) and (4) in Theorem, under the additional condition that the residue fields $A / M$ are infinite for all maximal ideals $M$ of $A$, proves the second assertion of the corollary.

\section{References}

[1] S. S. AbHYankar, Local rings of high embedding dimension, Amer. J. Math., 89 (1967), 1073-1077.

[2] D. KIRBY, The reduction number of a one-dimensional local ring, J. London Math. Soc., 10 (1975), 471-481.

[3] K. KuвотA, On stable ideals, Tokyo J. Math., 8 (1985), 449-454. 
[4] J. Lipman, Stable ideals and Arf rings, Amer. J. Math., 93 (1971), 649-685.

[5] E. MatLIs, The multiplicity and reduction number of a one-dimensional local ring, Proc. London Math. Soc., 26 (1973), 273-288.

[6] M. Nagata, Local Rings, Interscience, New York, 1962.

[7] M. NARITA, A note on the coefficients of Hilbert characteristic functions in semi-regular local rings, Proc. Cambridge Philos, Soc., 59 (1963), 269-275.

[8] D. G. NorthcotT, Prime ideals and Dedekind orders, Proc. London Math. Soc., 10 (1960), 480-496.

[9] D. G. NoRThCotT, A note on the coefficients of the abstract Hilbert function, J. London Math. Soc., 35 (1960), 209-214.

[10] D. G. NorthcotT and D. ReEs, Reductions of ideals in local rings, Proc. Cambridge Philos. Soc., 50 (1954), 145-158.

[11] J.D. SALLY, On the associated graded ring of a local Cohen-Macaulay ring, J. Math. Kyoto Univ., 17 (1977), 19-21.

[12] J.D. SALLY, Cohen-Macaulay local rings of maximal embedding dimension, J. Algebra, 56 (1979), 168-183.

[13] J. D. SAlly, Stretched Gorenstein rings, J. London Math. Soc., 20 (1979), 19-26.

[14] J. D. SAlly, Super-regular sequences, Pacific J. Math., 84 (1979), 465-481.

[15] G. Valla, On form rings which are Cohen-Macaulay, J. Algebra, 58 (1979), 247-250.

[16] O. Zariski and P. Samued, Commutative Algebra II, Van Nostrand, Princeton, N. J., 1960.

Present Address:

Department OF Mathematics

The National Defence ACademy

Yokosura 238 